\title{
Third Harmonic Generation in Silica Microfibres
}

\author{
Timothy Lee, Yongmin Jung, Christophe Codemard, Gilberto Brambilla \\ Optoelectronics Research Centre, University of Southampton, SO17 1BJ, United Kingdom. \\ tl305@orc.soton.ac.uk \\ Neil G. R. Broderick \\ Physics Department, University of Auckland, Private Bag 92019, New Zealand.
}

\begin{abstract}
We theoretically and experimentally study third harmonic generation in silica microfibres. Phase matching at critical diameters was achieved by intermodal-coupling with higher order third harmonic modes, which were successfully generated using $4 \mathrm{~ns}$ $1.55 \mu \mathrm{m}$ pump pulses.

(C) 2011 Optical Society of America

OCIS codes: (190.0190) Non-linear Optics; (190.2620) Harmonic generation and mixing; (190.4160) Multiharmonic generation; (190.4370) Nonlinear optics, fibers
\end{abstract}

\section{Introduction}

Research interest into microfibres has been driven by their unique optical properties arising from a combination of their wavelength-scale diameter and strong index contrast, which for silica can enhance the nonlinearity by over 100 times compared with standard telecom fibre [1] owing to the tight modal confinement.

This allows a wide variety of nonlinear effects to be studied at low powers, and recently our attention has been focused on third harmonic generation (THG) in microfibres. In a silica microfibre the fundamental mode at the pump frequency can have the same effective index ( $\mathrm{n}_{\text {eff }}$ ) as a higher order mode at the 3rd harmonic and thus be phasematched. For this to occur, the microfibre must have a particular diameter found by solving Maxwell's equations [2], such that the intermodal dispersion compensates for the waveguide and material dispersion. To date, there remains little literature on THG in silica microfibres, with the highest reported efficiency of $\eta=0.05 \%$ achieved using 30 fs pulses from a $1.25 \mu \mathrm{m}$ Cr:forsterite pump with a peak power of $10 \mathrm{~kW}$ [4]. This conversion was limited by poor modal overlap between the pump and TH modes with which the taper was designed to phase match with. In other reports, THG was achieved by coupling with the $H_{12}(3 \omega)$ mode, which experiences a greater overlap with the pump mode [3], and indeed significant UV power was detected when the taper was pumped with 30ns pulses from a $1.06 \mu \mathrm{m}$ $2 \mathrm{kHz}$ Nd:YAG laser. However, the conversion of $10^{-6}$ was low due to the short $100 \mu \mathrm{m}$ waist length.

Therefore, we report here on the modelling and experimental observation of THG in air clad silica microfibres when phase matched to the $H E_{12}(3 \omega)$ mode over much longer uniform waist distances of several millimetres. In addition, longer tapers up to $4.5 \mathrm{~cm}$ were used to study phase matching to other higher order TH modes.

\section{Theoretical Modelling}

Efficient third harmonic generation requires two conditions; the first is phase matching of the wavevectors, and the second is a large overlap integral between the modes. From symmetry conditions it can be shown that the overlap between the fundamental $H E_{11}(\omega)$ mode and the higher order $T E(3 \omega), T M(3 \omega)$ and hybrid $H E_{v m}(3 \omega)$ and $E H_{v m}(3 \omega)$ modes with even azimuthal order $v$ are identically zero - thus, we only consider the phase matching to hybrid modes with odd $v$ order. The corresponding microfibre diameters at which the phase matching condition $\mathrm{n}_{\text {eff }}(\omega)=\mathrm{n}_{\text {eff }}(3 \omega)$ is satisfied are given in Fig. 1. A pump wavelength of $1.55 \mu \mathrm{m}$ would be expected to phase match with the $H E_{12}(3 \omega)$ mode in a $0.77 \mu$ m diameter taper, and the $E H_{31}(3 \omega)$ mode in a $2.5 \mu \mathrm{m}$ taper. The former mode's overlap is the greatest, and by numerically solving the differential equations adapted from Ref. [2], the evolution of the third harmonic amplitude along the microfibre can be deduced as shown in Fig. 2 (a). The maximum theoretical conversion is $70 \%$ over $5 \mathrm{~cm}$, and when optimally detuned the amplitude grows monotonically with distance rather than varying sinusoidally.

Fig. 2 (b) shows that the TH amplitude resembles a sinc function of detuning, but is asymmetric about the peak and offset from zero by $-500 \mathrm{~m}^{-1}$ due to the influence of self and cross phase modulation. It is clear that efficient THG is only possible across a narrow range of detunings, and expressing the detuning as a function of the fibre diameter shows that to achieve $\eta>1 \%$ would require the diameter to be within $<1 \mathrm{~nm}$ from the critical diameter. With this in mind we proceeded to carefully taper the fibres with the results discussed below. 


\section{NTuD7.pdf}

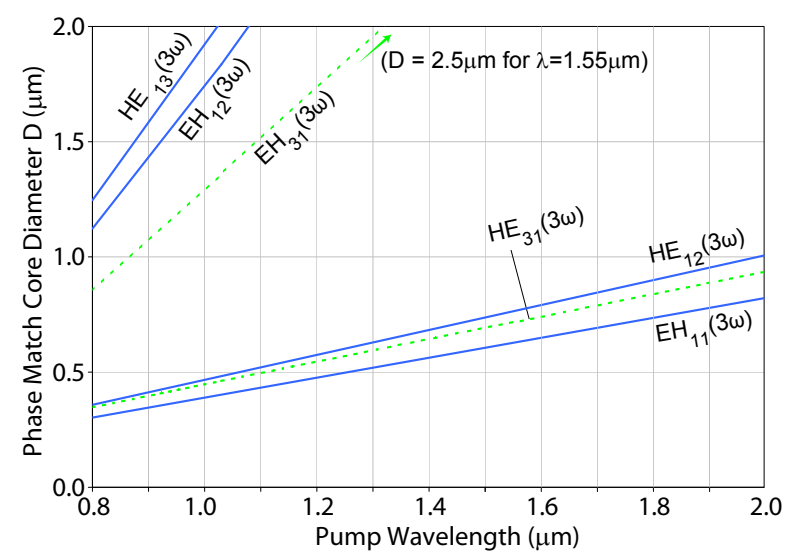

Fig. 1. Critical microfibre diameters for phase matching to TH modes at different pump wavelengths, when matched to $H E_{v, m}(3 \omega)$ and $E H_{v, m}(3 \omega)$ modes for $v=1$ (blue) and $v=3$ (green).
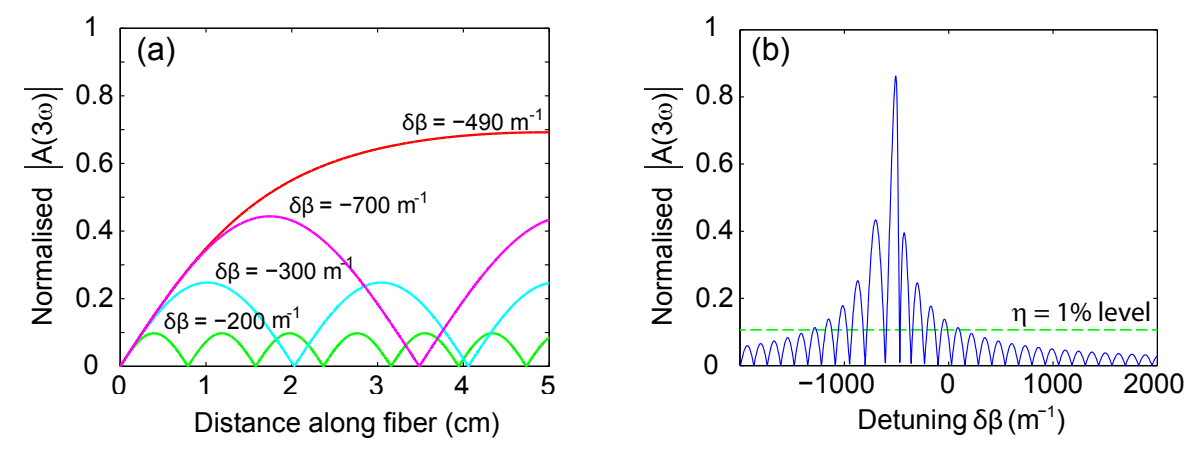

Fig. 2. Dependence of the third harmonic amplitude on (a) distance and (b) detuning, for a $1 \mathrm{~kW}$ $1.55 \mu$ m pump coupling to the $\mathrm{HE}_{21}(3 \omega)$ mode over a uniform $5 \mathrm{~cm}$ waist length.

\section{Experimental results}

Tapers were fabricated from standard single mode fibre using a rig in which the tensioned fibre is translated back and forth through a ceramic heater, which has a wide hot zone of $4 \mathrm{~mm}$ to ensure even heating. During the tapering process, the fibre is pumped with $4 \mathrm{~ns} 100 \mathrm{kHz}$ pulses from a fiberised $1.55 \mu \mathrm{m}$ laser and the output is connected to a shortpass filter before being measured on a spectrum analyser. The onset of THG can hence be easily detected so the rig is stopped once the critical diameter is attained. The moderately long pulse length minimises walkoff issues, and the high average and peak powers up to $0.5 \mathrm{~W}$ and $1.25 \mathrm{~kW}$, respectively, were well tolerated by the microfibre.

The results summarised in Table 1 show that the experimental efficiencies for the short tapers are an order of magnitude lower than the theoretical maximum, most likely due to discrepancy between the actual and critical diameter, but increase with length as expected. The maximum efficiency obtained with sample S3 was approximately $10^{-3}$, when pumped at $1.25 \mathrm{~kW}$ peak power $(10 \mu J$ pulse energy). A spectrum from $\mathrm{S} 1$ is given in Fig. 3 as an example, showing THG is significant even for peak powers down to $0.4 \mathrm{~kW}$. Other than minor spectral broadening, there do not appear to be any other major competing nonlinear effects. The experiments with longer tapers show that other TH modes (such as $E H_{31}(3 \omega)$ ), which were previously assumed to have poor overlap [2], are in fact capable of generating significant third harmonic power up to $\eta=10^{-4}$ for waist lengths of $3 \mathrm{~cm}$ or more.

\section{Conclusion}

The third harmonic phase match points and predicted efficiencies have been calculated for silica microfibres. Experimentally, 0.7-0.8 $\mu$ m diameter tapers with highly uniform waist regions of several millimetres were fabricated and demonstrated efficiencies close to $\eta \approx 10^{-3}$, which is comparable to the highest published value despite pumping 


\section{NTuD7.pdf}

with a tenfold smaller peak power. Future work will aim to optimise this efficiency further by extending the waist length whilst maintaining a tight diameter tolerance. Finally we note that, contrary to previously published work, in longer tapers significant THG can also be observed due to phase matching to other higher order modes.

The authors are grateful for insightful discussions and contributions from Konstantin Karapetyan, Prof. D. Meschede and Prof. A. Rauschenbeutel.

\begin{tabular}{ccccc}
\hline $\begin{array}{c}\text { Taper } \\
\text { Ref. }\end{array}$ & $\begin{array}{c}\text { Diameter } \\
(\boldsymbol{\mu} \boldsymbol{m})\end{array}$ & $\begin{array}{c}\text { Waist } \\
\text { Length }(\mathbf{m m})\end{array}$ & $\begin{array}{c}\eta \\
\text { (Measured) }\end{array}$ & $\begin{array}{c}\eta \\
\text { (Theory) }\end{array}$ \\
\hline S1 & 0.75 & $1 \pm 0.5$ & $5 \times 10^{-5}$ & $1 \times 10^{-3}$ \\
S2 & 0.75 & $2 \pm 0.5$ & $3 \times 10^{-4}$ & $5 \times 10^{-3}$ \\
S3 & 0.78 & $4 \pm 0.5$ & $\approx 10^{-3}$ & $2 \times 10^{-2}$ \\
\hline L1 & 1.8 & $45 \pm 0.5$ & $5 \times 10^{-5}$ & $4 \times 10^{-3}$ \\
L2 & 2.4 & $30 \pm 0.5$ & $1 \times 10^{-4}$ & $2 \times 10^{-3}$ \\
L3 & 2.6 & $45 \pm 0.5$ & $\approx 10^{-4}$ & $4 \times 10^{-3}$ \\
\hline
\end{tabular}

Table 1. Details for three short (S1-3) and long (L1-3) tapers, which are phase matched to the $H E_{12}(3 \omega)$ and $E H_{31}(3 \omega)$ third harmonic modes respectively. Diameter uncertainty: $\pm 0.1 \mu \mathrm{m}$.

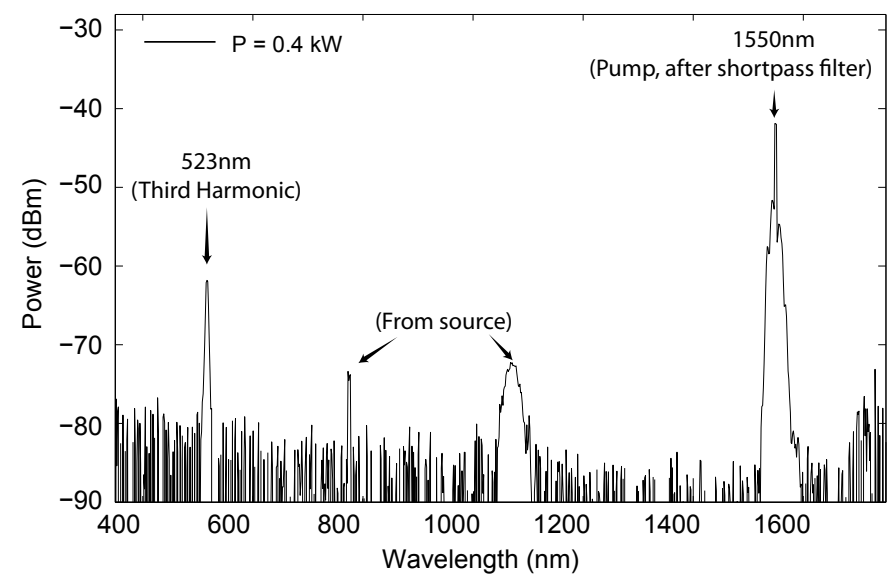

Fig. 3. Output spectrum for taper S1 showing third harmonic when pumped at $1550 \mathrm{~nm}$ with $0.4 \mathrm{~kW}$ peak pulse power, measured through a $1200 \mathrm{~nm}$ cutoff shortpass filter to attenuate the pump.

\section{References}

1. S. Afshar and T. M. Monro, "A full vectorial model for pulse propagation in emerging waveguides with subwavelength structures part I: Kerr nonlinearity," Opt. Expr. 17, 2298-2318 (2009).

2. V. Grubsky and A. Savchenko, "Glass micro-fibers for efficient third harmonic generation," Opt. Expr. 13, 6798-6806 (2006).

3. V. Grubsky and J. Feinberg, "Phase-matched third-harmonic UV generation using low-order modes in a glass micro-fiber," Opt. Comm. 274, 447-450 (2007).

4. D. Akimov, A. Ivanov, A. Naumov, O. Kolevatova, M. Alfimov, T. Birks, W.Wadsworth, P. Russell, A. Podshivalov, and A. Zheltikov, "Generation of a spectrally asymmetric third harmonic with unamplified 30-fs Cr: forsterite laser pulses in a tapered fiber," App. Phy. B: Lasers and Optics 76, 515-519 (2003).

5. U. Wiedemann, K. Karapetyan, C. Dan, D. Pritzkau, W. Alt, S. Irsen, and D. Meschede, "Measurement of submicrometre diameters of tapered optical fibres using harmonic generation," Opt. Expr. 18, 7693-7704 (2010). 